\title{
Cost Analysis of a Commercial Manufacturing Process of a Fine Chemical Compound Using Micro Process Engineering
}

\author{
Ulrich Krtschila, Volker Hessel ${ }^{\star a c}$, Dana Kralisch ${ }^{d}$, Günther Kreisel ${ }^{d}$, Michael Küpper ${ }^{b}$, \\ and Rainer Schenk ${ }^{b}$
}

\begin{abstract}
In the last years micro process engineering has developed rapidly and commercial interest has increased. While process intensification was demonstrated, the question remains whether this novel technology can lead to economic profits. In this context, a cost analysis is performed here for an economic fine chemical process of the customized chemical producer AzurChem $\mathrm{GmbH}$, i.e. the formation of the 4-cyanophenylboronic acid, using the benefits of micro process technology supplied by IMM GmbH. This process is representative for several other fine and specialty chemical manufacturing processes proprietary to the AzurChem $\mathrm{GmbH}$. This is the first time that a cost analysis is made accessible in open literature for a commercial chemical product made by micro process engineering, i.e. for a real-case scenario with validated database, i.e. real yields, micro-chemical plant, chemical and operator costs. The conclusions from this particular case are extended to more general statements on the micro processing of the whole class of high-value fine chemicals. This is achieved via some potential case scenarios of conceivable improvements by means of capacity or selectivity increases and a comparison with a traditional manufacturing method based on batch processing. In this way, some fundamental issues on the suitability of this novel technology are revealed, i.e. how process intensification translates into business drivers.
\end{abstract}

Keywords: Cost analysis · 4-Cyanophenylboronic acid $\cdot$ Micro process engineering $\cdot$ Micro reactor · Microstructured reactor

${ }^{*}$ Correspondence: Prof. Dr. V. Hessel

Tel.: +496131990450

Fax: +496131990205

E-Mail: hessel@imm-mainz.de

alnstitut für Mikrotechnik Mainz $\mathrm{GmbH}$

Carl-Zeiss-Strasse 18-20

D-55129 Mainz

www.imm-mainz de

${ }^{\mathrm{b}} \mathrm{AzurChem} \mathrm{GmbH}$

Carl-Zeiss-Strasse 18-20

D-55129 Mainz

E-Mail: info@azurchem.com

www.azurchem.com

'Eindhoven University of Technology

Chemical Engineering \& Chemistry Department

Den Dolech 2

NL-5612 AZ Eindhoven

dFriedrich-Schiller-University Jena

Institut für Technische Chemie und Umweltchemie

Lessingstr. 12

D-07743 Jena

www.ituc.uni-jena.de

\section{Introduction}

There is now overwhelming evidence that microstructured reactors and micro process engineering are highly promising valuable novel tools and processing approaches to enable process intensification, shared with further reactor and process engineering advantages such as process safety, legislation, or modularity. Industry currently implements these new ideas into business. Most industrial studies, if published at all, relate to pilot studies, touching upon the engineering applicability of micro process engineering. Recent statements of industrial leaders and magazine reviews leave hardly any doubt anymore that the technology is going to be used [1][2]. This intrinsically implies that there are business drivers for doing so and that cost analyses were performed internally in the chemical industry. With two exceptions, however, such knowledge is not disclosed. One of these is a study from the fine chemical and pharmaceutical company Lonza, Visp/Switzerland, detailing capital (CAPEX) and operational (OPEX) costs for several pilot microchemical processes [3]. No advantages for the CAPEX costs were determined, because the microstructured reactors were as or more costly than the equipment routinely used, which are glass-lined vessels with impellers. In contrast, the OPEX costs were significantly improved and this was specified to the different portions such as labor, plant costs, QA/QC, waste treatment, transport \& logistics, and change over \& cleaning. Overall, it was stated that the OPEX improvement outpaced the additional CAPEX costs, but the ratio between the gains and additional costs was not given and thus no net profit can be deduced [3]. Merck, Darmstadt/Germany, made together with the Technical University Clausthal a four-staged potential analysis, which started with a technological evaluation (theoretical and technical potential) to come finally to a business view (material and economical potential) [4]. For the latter, data on profitability and amortization time were given. A conclu- 
sion of whether the micro-chemical process is better than conventional routes and if it will be applied is not given. The chemical process investigated was nitration to yield 3-methyl-4-amido-5-nitro benzoic acid ester. Besides these two reports, a German project ( $\mu$ VTGuide, funded by BMBF, [5]) has been initiated where the chemistry organization Dechema and the chemical company BASF are going to evaluate the potential of chemical micro process engineering based on BASF's widely developed eco-efficiency analysis [6]. Jena University has applied life-cycle assessment analysis (LCA, [7]) to a micro-chemical process, the Li-based synthesis of $m$-anisaldehyde from $n$-bromoanisole, at laboratory scale [8] and recently reported on the same investigation for the production scale for the same process [9]. Benefits in terms of cumulative energy demand (CED) as well as green house effect, acidity, and toxicity potentials were outlined [8]. An exergy analysis (see [10-12]) was made for microstructured fuel processor technology [13] and a generic benchmarking of microstructured catalytic reactors [14] was given, with the catalyst and overall reactor volume as the figures of merit.

Thus, the investigation of the economic potential of chemical micro processing has begun. However, a generic view is still missing, i.e. which type of microstructured reactors and which type of plants are suited for which type of chemical processing and what are the key figures to optimize micro-chemical processes. Since micro process technology is for many reasons still far away from the day-to-day experience of industry's process engineers, the performance of a generic cost analysis at this point of development is not simply the mechanical repetition of the same automatism for conventional chemical processes (see e.g. [15] for the cost analysis method); it rather gives scientific insight how applied developments should orient on in future.

Moreover, a cost analysis for micro chemical processing is performed here for the first time based on a commercial process and product, while the abovementioned studies refer to pilot processes under further optimization, as far as information is disclosed. AzurChem GmbH, a spin-off of the Institut für Mikrotechnik Mainz GmbH (IMM), produces and sells fine and specialty chemicals, manufactured with its own and IMM hardware, for researchers, developers and producers in chemistry and biotechnology.

In the following, a cost analysis was done regarding a real existing process and the results were then compared with some scenarios based on different and partly virtual assumptions.

\section{Cost Calculation Methodology, Chemical Process, and Case Studies}

\subsection{Cost Calculation Database and Shares Considered}

Significant revenue shares for finechemical plants refer to raw-material supply, waste disposal, operator salaries, and finally the investment of the plant itself. Accordingly, the cost analysis includes both fixed costs and variable costs, roughly corresponding to the CAPEX and OPEX costs, respectively.

\subsubsection{Variable Costs}

The variable costs include costs for reagents, the operator's salary, energy consumption, and disposal. The cost figures for the reactant, the metallization agent, the borate, the solvent and some adjuvant used as input for the calculation comply with the purchase prices of AzurChem GmbH for one production period, in which $10 \mathrm{~kg}$ 4-cyanophenylboronic acid are manufactured. For some scenarios these costs were adapted as described there.

For the calculation of the operator's salary it is assumed that the supervision of the continuous working reaction plant can be done in parallel to the purification steps of the crude 4-cyanophenylboronic acid that takes place batch-wise. In the case of the existing process the overall time allocation for plant operation is obtained from AzurChem $\mathrm{GmbH}$, in the other cases it follows industrial practice as far as known from discussion with industrial processing and cost analysis experts via personal communications [16] The salary used for the calculations is based on costs for skilled German personnel. Due to the small dimensions the micro reaction plant can be started up and shut down in a short time. A daily working time of eight hours, five days a week and 50 weeks per year is the basis of the calculation.

The energy costs are mainly limited to the electric power consumption of the thermostats and the pumps. Average energy costs of $0.25 € / \mathrm{kWh}$ are used in the cost analysis. Disposal costs for the waste, especially for the halogen-containing solvent, are based on the specific costs incurred by AzurChem $\mathrm{GmbH}$. In a second step all the variable costs were related to $1 \mathrm{~kg}$ of the purified and saleable product.

\subsubsection{Fixed Costs}

The method used is a simplified analysis of fixed costs absorption.

\section{Product Related Fixed Costs}

The equipment costs encompass both the existing micro reaction plant according to Fig. 1, including three microstructured reactors, four pumps, valves and piping, measurement and control technology, cryostat and installation costs and the necessary devices for the purification, e.g. a distillation unit. These costs had to be divided by the depreciable life and then are summarized with the annual maintenance costs and the annual costs for premises. Based on the fact that premises costs only have a marginal effect on the product-related fixed costs they were obtained from estimated costs of $300 €$ for a laboratory per year and square meter multiplied with the required $3 \mathrm{~m}^{2}$ floor space for micro reactor processing.

In accordance with AzurChem GmbH's practice an amortization period of five years was assumed.

\section{Remaining Fixed Costs}

Including the remaining fixed costs in the total cost evaluation is necessary to be sure that costs which arise that are not directly related to a specific product are accounted for. The remaining fixed costs include administration costs, costs for offices and sales activities, for instance. In the case considered here such costs are unknown in detail, but as a realistic approach an overhead in terms of $50 \%$ of the variable and product related fixed costs was added.

\subsection{Chemical Reaction Investigated}

Boronic acids are used as intermediates for the synthesis of pharmaceuticals and fine chemicals, e.g. for Suzuki couplings. High-value boronic acids are within the product portfolio of AzurChem GmbH besides other precious fine chemicals. As representative of this class of chemical intermediates, the manufacturing process of the 4-cyanophenylboronic acid (Scheme) was chosen to be investigated.

One key feature of the reaction is the high price of the raw materials and respectively of the chemical product 4-cyanophenylboronic acid; therefore the process is dominated by the high-value raw materials.

\subsection{Process Flow and Plant}

Fig. 1 shows a basic flow sheet of the investigated synthesis process and the used continuous working micro reaction plant. Four pumps deliver the three reactants and the quenching substance. The main reactant is primarily dissolved in a certain amount

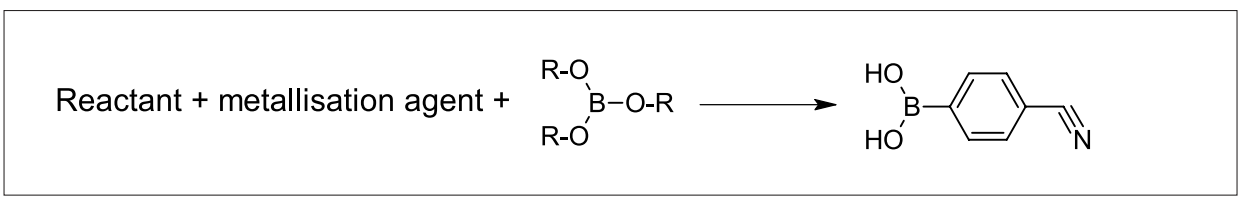

Scheme. 


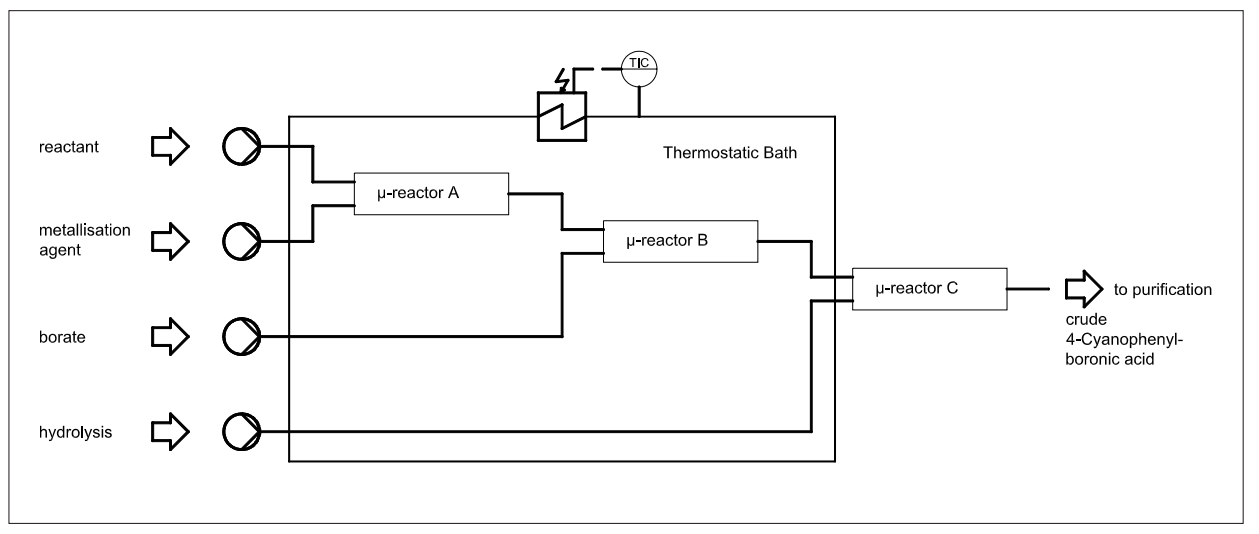

Fig. 1. Simplified flow sheet of the reaction plant

of a solvent and reacts with a metallization agent in microstructured reactor A. Afterwards the resulting product reacts with a borate in microstructured reactor B mainly to the final product 4-cyanophenylboronic acid. Both reactors are immersed in a thermostatic bath. In a last step the reaction is quenched by hydrolysis in micro reactor $\mathrm{C}$. The subsequent purification steps like distillation are performed batchwise.

\subsection{Choice of Case Studies}

Microstructured reactors can improve selectivity and in this way reduce the costs for chemical starting materials, waste disposal, and energy [17]. This can sometimes be achieved by simply transferring a batch protocol into a continuous flow micro reactor operation with increased mass and heat transfer and kinetically derived (shorter) residence times. The latter may also impact the operator costs. In many other cases, however, the simple repetition of batch processing protocols is not enough; process intensification demands new tailored protocols for chemical micro process engineering, termed 'novel chemistry' [18]. Both cases are considered here.

A virtual case for achieving higher selectivity was added. An improvement gap of $20 \%$ was added, based on reported selectivity achievements of Clariant [19] and Merck [20] for organometallic reactions.

One prominent scale-out concept for microstructured reactors is 'numbering-up' (see e.g. [17]). Here, the concept of external numbering-up of ten microdevices in parallel was considered, being a realistic number based on our expertise. Therefore, a virtual case such as this was added to the cost analysis.

Another scale-out concept is 'smart dimensioning', i.e. a small increase in characteristic internal dimensions without losing performance [21]. This is practiced especially for mixer-reactors using many grouped-class devices, dedicated to IMM's chemical processing concepts. The same, however, can be done by process intensifica- tion via 'novel chemistry' [18]). In this way, order-of-magnitude changes in productivity are achieved (see reported examples, e.g. in [17][18][22-25]) which, respectively, also may decrease the plant size per given production rate, targeting the operator salaries and the plant investments. This is achieved by not only exploiting the engineering potential of microstructured reactors, but by using the latter to utilize essentially 'novel organic chemistry' e.g. at high temperatures combined with high pressures combined with very short residence times [18]. Again, a virtual case such as this was considered in two variants, assuming five- and ten-fold increases in productivity.

\section{Results and Discussion}

These calculations are based on an average yield of the process of $75 \%$, including not only the reaction yield but also the loss of the product within the purification process.

\subsubsection{Cost Allocation of the Manufacturing Costs}

Fig. 2 illustrates the large share of variable costs amounting to $63 \%$ compared with the product-related fixed costs of only

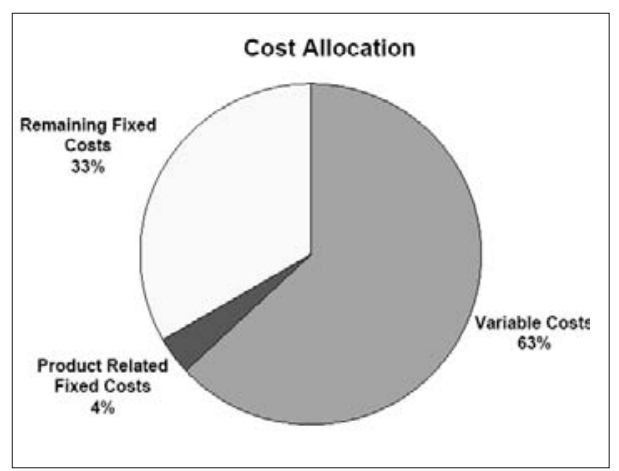

Fig. 2: Cost allocation of the existing process

\subsection{Cost Analysis of the Existing Micro-chemical Process}

$4 \%$. This is caused by the use of high-value fine chemical raw materials and by the large share of operator costs for any chemical process (at least when based on German salary standards, as is the case here). The investment costs for micro process equipment thus cannot be a major decision driver in this case, whereas the importance of suitable micro process engineering (also for future process optimization) is evident, affecting directly the variable costs. This is in accordance to other fine-chemical studies done by some of the investigators, used for internal purposes or to be published [26], and thus seems to be general for finechemical syntheses with microstructured reactors. These studies also demonstrate that the costs of the microstructured reactors usually amount to less than $10 \%$ of the overall plant-related costs (not published here, see [26]), which go along with the costs of conventional plant engineering. Therefore, the microstructured reactor costs have nearly no relevance for the overall decision for or against this new technology, but rather their performance and reliability are main drivers (future studies may consider plant breakdown). In this context, it has to be mentioned that the costs of today's microstructured reactors and their plants may vary largely, for example off-the-shelf devices integrated into conventional balance-of-plant equipment to give retrofitted plants compared to customized, complex (e.g. highly integrated) devices within specialized peripherals and in-house developed plant interconnection. The prices may vary by a factor of ten and influence strongly the product-related costs. The fraction of the remaining fixed costs here comprises one third of the total costs, which however is given by definition (see Section 2.2.2).

\subsubsection{Variable Costs}

There are two major constituents of the variable costs which represent together almost $98 \%$ (Fig. 3). The costs of the starting substances comprise $65.8 \%$ and the salary for the operator is $32.1 \%$. This as a net result, as mentioned above, is in accordance with results of other cost analyses we car-

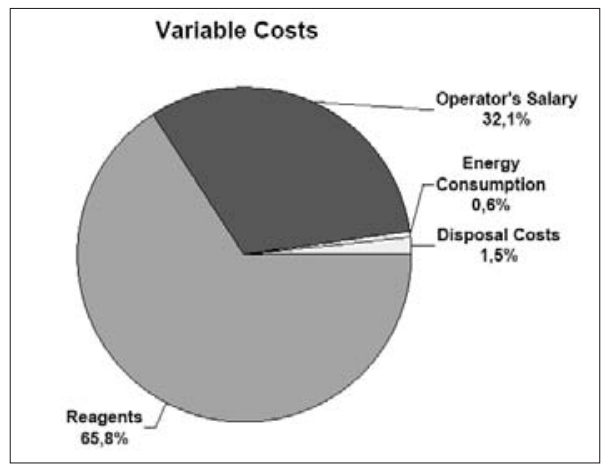

Fig. 3: Breakdown of the variable costs of the existing process 
ried out before [26]. However, the ratio between both cost portions is specific for the case considered here. Compared to the prior studies with average-priced fine chemicals this ratio tends to be inverted owing to the processing of high-cost materials. Indeed, the manufacturing of high-value chemicals in relatively small amounts requires a relevant operation effort. This also emphasizes that it is economical to produce such materials with micro process technology even in countries with a high wage level. The small fractions of energy consumption and disposal costs show that the use of the micro reaction technology leads to a relatively low environmental impact.

As the cost analysis of the existing manufacturing process of the 4-cyanophenyl-boronic acid points out, this can be done with a profit when using a continuous working micro reaction plant.

\subsection{Cost Analysis of a Virtual Batch Process}

A direct comparison with a real existing batch process is not possible, since the synthesis route used in the micro reaction plant is not feasible in a batch reactor. For this reason a virtual batch reaction process was investigated using the same substances but with some reasonable assumed changes with respect to the micro-chemical process.

\subsubsection{Cost Allocation of a Virtual Batch Process}

To produce the same amount of a product in a certain time a batch process needs larger equipment compared to a continuous process. The reason is that such a process usually needs at least several hours and the reactor, feed vessels and receivers must be dimensioned to contain the necessary amounts. A significantly higher amount (calculated with a factor two) of the solvent was assumed to be necessary to dissipate the reaction heat. As a result the productrelated fixed costs fraction is a factor 1.5 higher compared with the existing microchemical process (Fig. 4).

\subsubsection{Variable Costs}

The higher amounts of solvent required lead to a doubling of the disposal costs fraction (Fig. 4). Furthermore, a batch process normally requires more attention from the operator and more costly process control equipment. In the calculation used here this leads to manufacturing costs even slightly higher than the sales price AzurChem $\mathrm{GmbH}$ could get for its product.

\subsection{Case Scenarios of Potential Improvements and their Influence on the Manufacturing Costs}

The motivation for selection of this and the following virtual case studies are

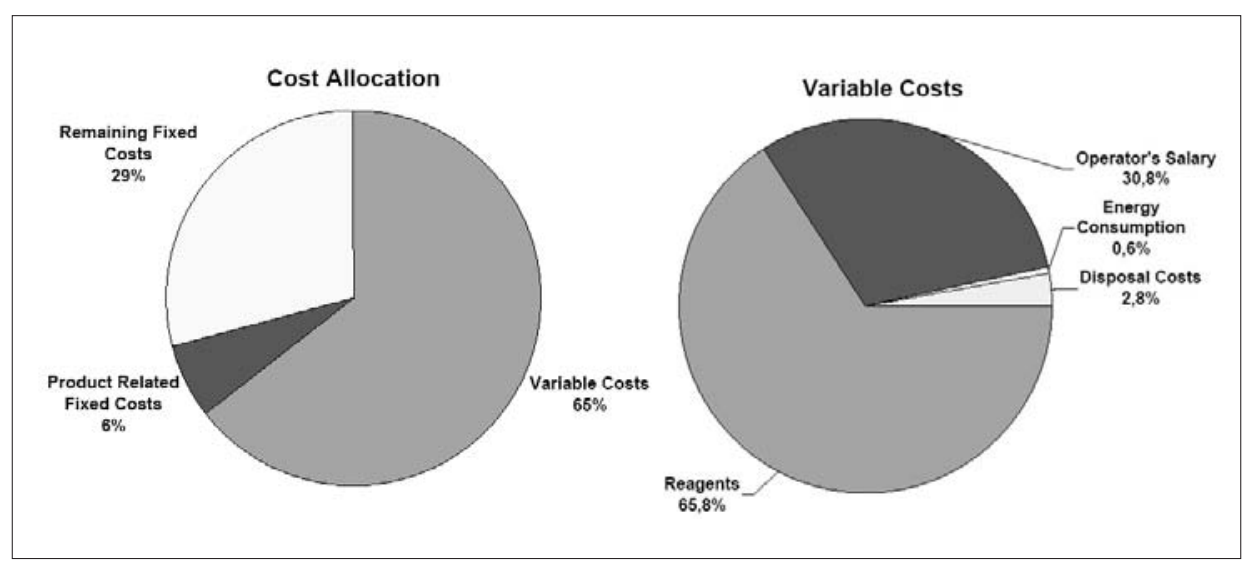

Fig. 4: Total cost allocation of a virtual batch process and shares of variable costs

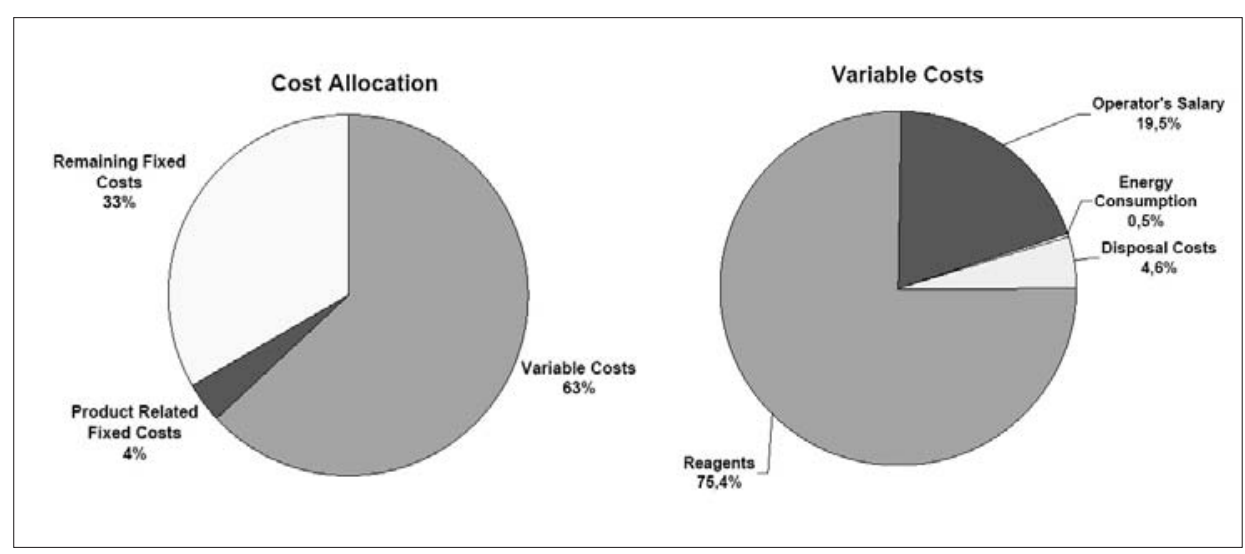

Fig. 5: Total cost allocation for fivefold capacity and share of variable costs

given in Section 3.5 (see above). At first, capacity increase is considered. This can be reached by so-called 'external numbering-up', which means a multiplication of the microdevices themselves as well as by an enlargement of the internal dimensions within a certain range. Concerning the latter, IMM has developed families of microstructured devices by scaling up and down the internal dimensions and has established some correlations of their performance, e.g. concerning the mixing times (see e.g. [27] for the StarLam mixer series). Here, micron-sized dimensions are given within the microstructured reactors ('micro-inside'), while the outer dimensions range from fist- to shoebox-size (and will even approach the meter-scale in near future).

\subsection{Microstructured Reactors with a Fivefold Higher Throughput}

This scenario assumes an averaged $20 \%$ reduction of the purchase prices of the reagents if a microstructured reactor with fivefold capacity would be used (Fig. 5). Compared with the existing process no notable differences in the allocation of the manufacturing costs can be observed, which as to be expected due to the very low contribution of the costs of equipment in gen- eral. However, regarding the variable costs, changes in the ratio between several factors are noted. While the operator's salary portion decreases from $32.1 \%$ to $19.5 \%$, an increase of the portion of the reagent costs is given and correlated with an increase in the disposal costs as well.

\subsection{Microstructured Reactors with a Tenfold Higher Throughput}

In this case the reagents costs were calculated with an averaged reduction of $25 \%$ when converting a tenfold amount (Fig. 6). A modification in the range of $2 \%$ lower product-related fixed costs and higher variable costs owing to a further decrease of the operator's salary portion down to $12.6 \%$. Conversely, the fractions of reagent costs and waste disposal rise to $81.1 \%$ and $6 \%$, respectively.

\subsection{Ten Microstructured Reactor Lines in Parallel}

The assumptions regarding to the reagent costs are the same as in the case of a tenfold reactor capacity (Fig. 7). Since the tenfold multiplication of the micro reactor costs leads to significantly higher investment costs it is not surprising that the product-related fixed costs fraction doubles compared with the existing plant. 


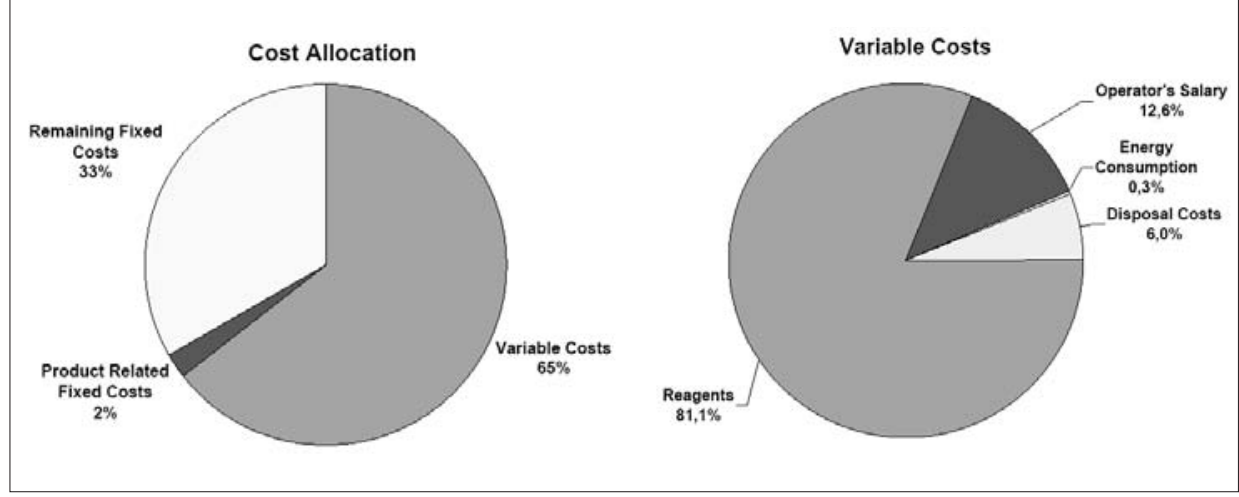

Fig. 6: Total cost allocation with tenfold capacity and variable costs

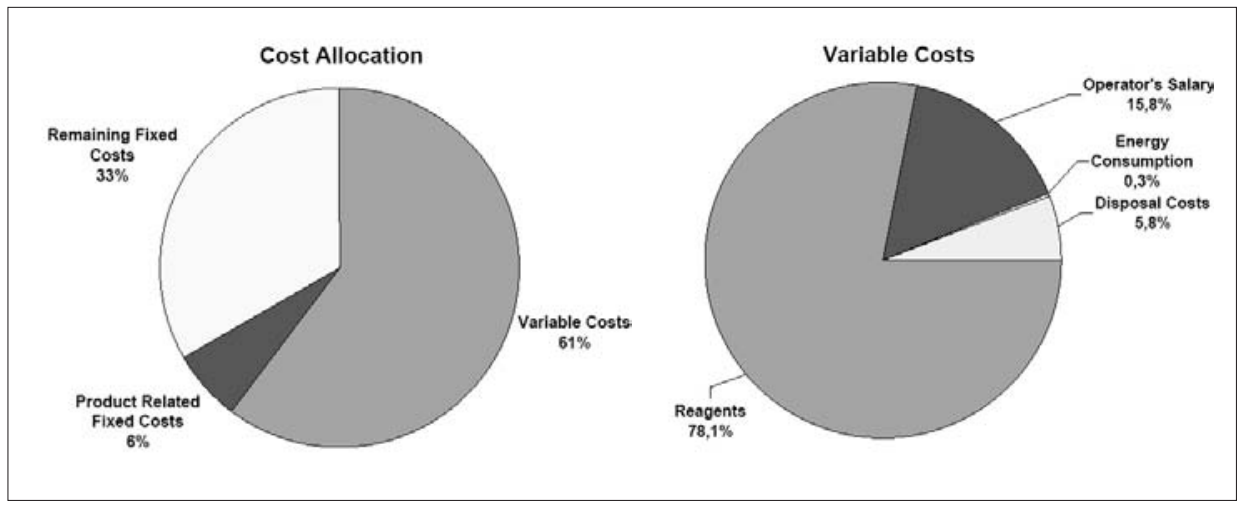

Fig. 7: Total cost allocation for ten microstructured reactor lines and variable costs

Assuming that the handling of ten reactor lines in parallel is about $30 \%$ more timeconsuming than for a single one, the portion of the operator's salary is almost 3\% higher compared with the tenfold capacity of only one micro reactor line.

\subsection{Optimized Process with Higher Yield}

The relevance of selectivity increase (to give higher yield) as one main motivation in the process optimization studies is investigated for the case studied here. In many practical cases, however, the manufacturing process for a product which is produced temporarily and in relatively small amounts is only optimized to a certain extent for economic reasons. Further investigations thus depend on the trade-off between higher earnings and the costs of such investigations.

Selectivity improvements may result from the reaction itself or from less product loss during purification. The influence of a $5 \%$ yield increase on the relation between costs and earnings is shown in Fig.
8 . The change is about $3 \%$ which is 'nice to have', but normally does not justify cost-intensive studies.

\subsection{Comparison of Total Costs for all Scenarios - Profitability}

The total costs are highest for the virtual batch and amount to $133 \%$, when leveling the total costs of the real microchemical process to $100 \%$ (Fig. 9). For the three different scenarios with increased capacity a dramatic decline in costs can be achieved. The total costs are reduced to one third (33\%) for the micro-chemical process with fivefold capacity and further decline to $25 \%$ in the case of tenfold higher capacity, respectively. It is evident that both process intensification and numbering-up provide a practicable way to further increase profitability.

A similar view is provided by the comparison of the ratios between manufacturing costs and earnings (Fig. 10). For all scenarios considered the theoretically attainable selling price was reduced to the same degree as the purchase prices were assumed. Under more realistic conditions of future competition, the selling price for such relatively large amounts of the product will probably fall even further. Similar to the results given in Fig. 9, Fig. 10 also highlights possible cost advantages by using microstructured reactors with higher throughput, presuming the produced amounts can be sold on the market.

\subsection{Comparison of Costs for In- vestments (Product-related Fix Costs)}

The costs for the investments into equipment (microstructured reactors and balance-of-plant equipment) are small compared to the total costs, as mentioned before. Nonetheless, since the purchase of this equipment is one of the first steps when entering process development and

\section{Influence of the yield to costs and earnings}

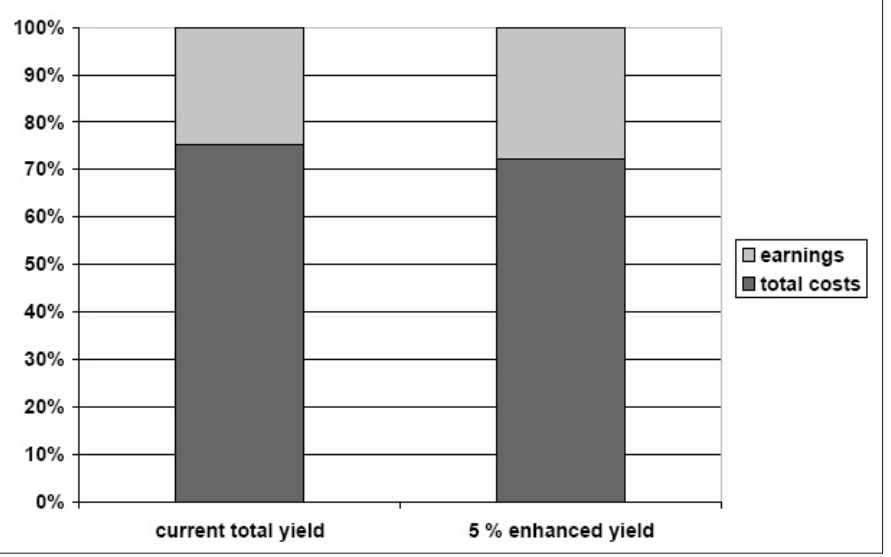

Fig. 8: Influence of the yield to costs and earnings

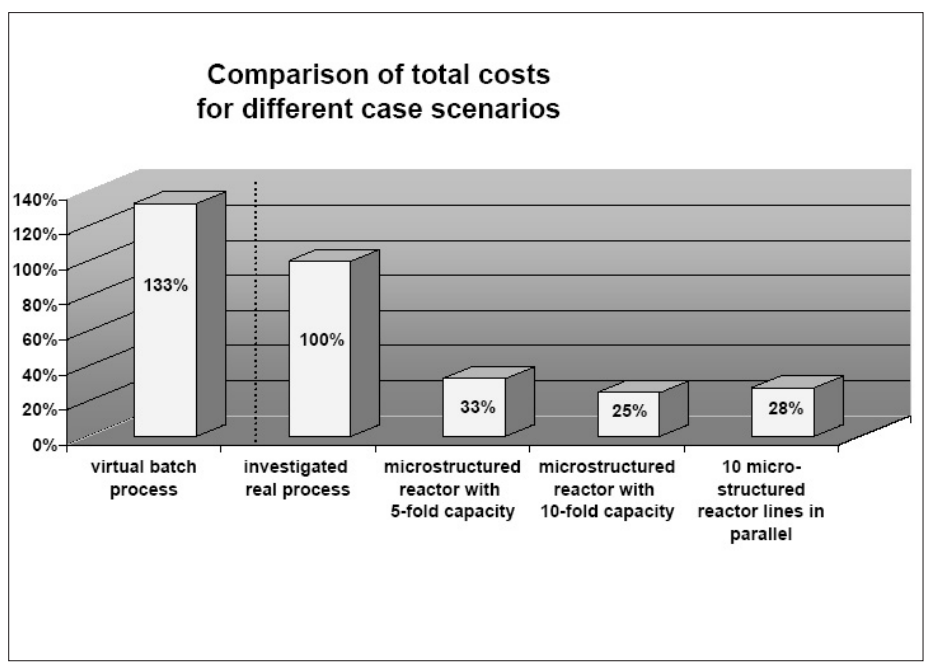

Fig. 9: Comparison of total cost for different case scenarios 


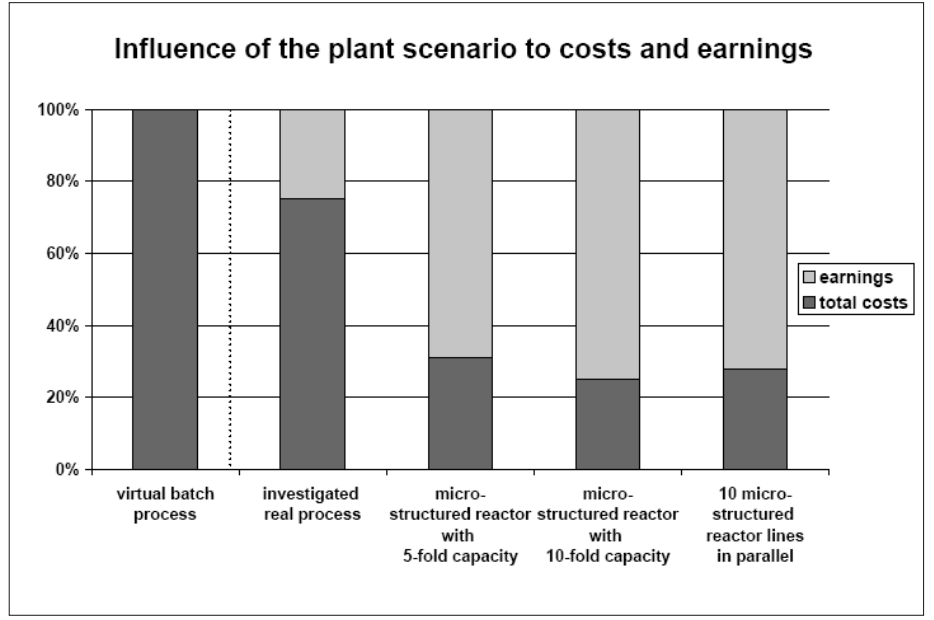

Fig. 10. Influence of the plant scenario to costs and earnings

plant installation, the corresponding costs may impact the final decision in favor of or against the micro-chemical process. Fig. 11 provides a comparison of the share of the product-related fix costs which relates to the absolute equipment costs. The equipment costs are normalized to the possible earnings and are also given as total costs normalized to $100 \%$ for the real-case scenario. It is evident that process intensification (still with only one microstructured reactor) is a practicable way to reduce equipment costs. The earning-rated contribution declines to $2 \%$ and is the best option from the financial side, however, at the expense of further process development. Numbering-up increases equipment costs, but not to the same extent as the number of parallel operated microstructured reactors increase, from only $4 \%$ ('real process') to $6 \%$ ('ten micro reactor lines in parallel').

\section{Summary and Conclusions}

The cost analysis of the commercially applied manufacturing process for the fine chemical 4-cyanophenylboronic acid
(Fig. 12) points out that an optimization of operational (variable) costs is the key driver to develop a business perspective for micro process engineering. Two major trends are visible. The first relies on the synthesis of high-value products from expensive raw materials and the process mentioned above falls into this category. In such a case even the high operator costs, which otherwise dominate, are outpaced. The second strategy is based on reducing the operator costs by process intensification through micro process engineering as compared to the batch, which is also practiced and will be reported elsewhere [26].

In both cases, the equipment costs (microstructured reactors and balance-ofplant equipment) take up a small fraction of the total costs. Thus, ultimately these costs should have a minor impact on the decision to go for the novel technology, but rather the latter has to prove the expected process optimization and reliability.

Where demanded, an increase of capacity by numbering-up, smart scale-out or further process intensification concepts helps to further reduce the overall costs of the processes (in the case considered here down to about $25 \%$ ) and increases respec-

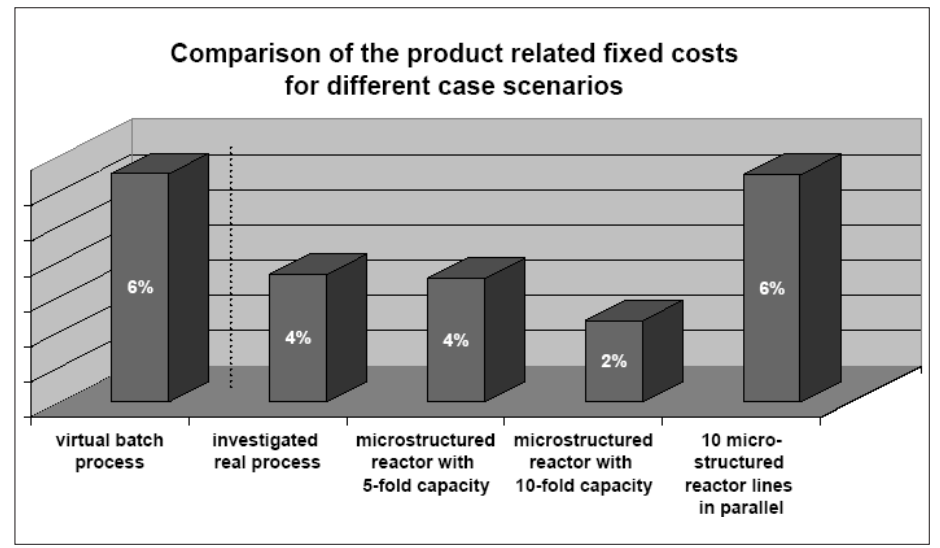

Fig. 11. Comparison of costs for investments (product-related fix costs)

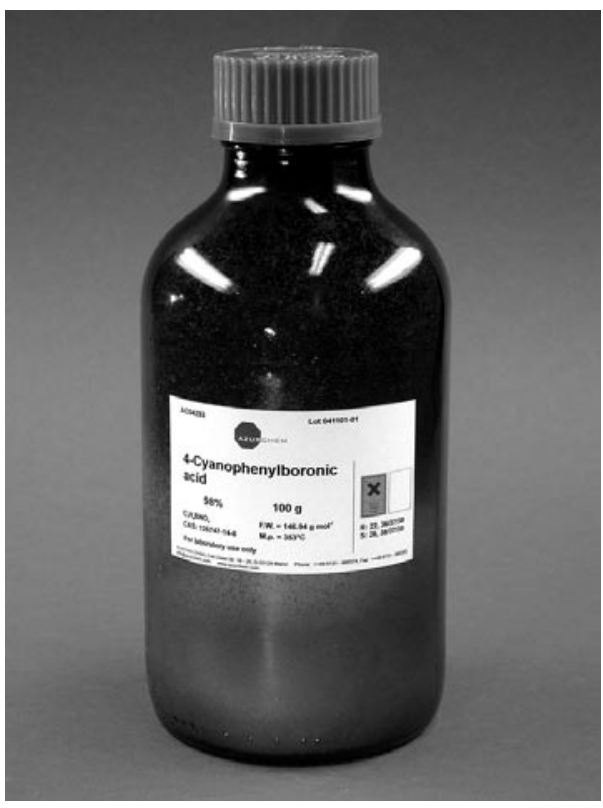

Fig. 12. Commercial product of AzurChem $\mathrm{GmbH}$, 4-cyanophenylboronic acid, made by micro process technology

tively the earnings. Thus, plants with microstructured reactors are more profitable when operated at larger scale, as their conventional counterparts.

Within the - certainly severe - limits of comparing a real-case micro-chemical and a virtual batch process, the respective cost comparison identified commercial advantages for the continuous synthesis in the micro reaction plant.

Finally, a cost analysis was analyzed for a real-case fine chemical process based on micro process technology for the first time. The fact that the results show earnings by the new path and - even more convincing - that the product is actually sold, make evident that micro process engineering is for some cases a commercially viable processing alternative.

Received: June 21, 2006

[1] S.-R. Deibel, CHEManager 2006, 2.

[2] A. Belloni, Process 2006, 13, 64 .

[3] D.M. Roberge, L. Ducry, N. Bieler, P. Cretton, B. Zimmermann, Chem. Eng. Tech. 2005, 28, 318.

[4] D. Schmalz, M. Häberl, N. Oldenburg, M. Grund, H. Muntermann, U. Kunz, CIT 2005, 77, 859 .

[5] BMBF Project $\mu$ VTGuide (Dechema, BASF), started 2005, guided by VDI/ VDE, Berlin, www.mstonline.de/foerderung/projektliste, search for ' $\mu$ VTGuide'.

[6] P. Saling, A. Kicherer, B. Dittrich-Kramer, R. Wittlinger, W. Zombik, I. Schmidt, W. Schrott, S. Schmidt, Int. J. LCA 2002, 7 , 203.

[7] A. Burgess, D. J. Brennan, Chem. Eng. Sci. 2001, 56, 2589.

[8] D. Kralisch, K. Kreisel, Chem. Ing. Tech. 2005, 77, 62 . 
[9] D. Kralisch, K. Kreisel, in 'AIChE Topical Conf. Proceedings', AIChE Spring Conference, Orlando/USA, April 26-30, 2006, published on CD.

[10] R.U. Ayres, L.W. Ayres, K. Martinás, Energy 1998, 23, 355.

[11] T.J. Kotas, Chem. Eng. Res. Dev. 1986, 64, 212.

[12] R. Riveiro, Energy Conversion and Management 2002, 43,1199.

[13] E.R. Delsman, C.U. Uju, M.H.J.M. de Croon, J.C. Schouten, K.J. Ptasinski, Energy 2006, in press.

[14] E.R. Delsman, M.H.J.M. de Croon, A. Pierik, G.J. Kramer, P.D. Cobden, C. Hofmann, V. Cominos, J.C. Schouten, Chem. Eng. Sci. 2004, 59, 4795.

[15] D. Hunkeler, G. Rebitzer, Int J LCA $\mathbf{2 0 0 3 ,}$ 8, 109.

[16] Personal communications from representatives from diverse medium and largesized chemical companies in the field (2006).

[17] V. Hessel, S. Hardt, H. Löwe, 'Chemical Micro Process Engineering - Fundamentals, Modelling and Reactions', WileyVCH, Weinheim, 2004

[18] V. Hessel, P. Löb, H. Löwe, Curr. Org. Chem. 2005, 9, 765.
[19] V.Hessel, C.Hofmann, H. Löwe, A. Meudt, S. Scherer, F. Schönfeld, B. Werner, Org. Proc. Res. Dev. 2004, 8, 511.

[20] H. Krummradt, U. Kopp, J. Stoldt, in 'Microreaction Technology: 3rd International Conference on Microreaction Technology, Proc. of IMRET 3', Springer-Verlag, Berlin, 2000, p. 181.

[21] V. Hessel, P. Löb, H. Löwe, in 'Proceedings of 4th International Workshop on Micro Chemical Plants', Ed.: Y.-I. Yoshida, Kyoto/Japan, January 26-27, 2006.

[22] S.T. Haswell, P. Watts, Green Chemistry 2003, 5, 240.

[23] K. Jähnisch, V. Hessel, H. Löwe, M. Baerns, Angew. Chem. Int. Ed. 2004, 43, 406.

[24] H. Pennemann, P. Watts, S. Haswell, V. Hessel, H. Löwe, Org. Proc. Res. Dev. 2004, 8, 422.

[25] V. Hessel, H. Löwe, Chem. Eng. Tech. 2005, 28, 267.

[26] V. Hessel, D. Kralisch, G. Kreisel, U. Krtschil, P. Löb, H. Löwe, Org. Proc. Res. Dev. 2006, to be submitted.

[27] B. Werner, V. Hessel, P. Löb, Chem. Eng. Tech. 2005, 8, 401. 